\title{
Asymptotics of Smallest Component Sizes in Decomposable Combinatorial Structures of Alg-Log Type
}

\author{
Li Dong, Zhicheng Gao, Daniel Panario ${ }^{1}$ and Bruce Richmond ${ }^{2}$ \\ ${ }^{1}$ School of Mathematics and Statistics, Carleton University, Ottawa, Canada \\ ${ }^{2}$ Department of Combinatorics and Optimization, University of Waterloo, Waterloo, Canada
}

received March 28, 2009, revised Jan 25, 2010, accepted Feb 3, 2010.

A decomposable combinatorial structure consists of simpler objects called components which by themselves can not be further decomposed. We focus on the multi-set construction where the component generating function $C(z)$ is of alg-log type, that is, $C(z)$ behaves like

$$
c+d(1-z / \rho)^{\alpha}\left(\ln \frac{1}{1-z / \rho}\right)^{\beta}(1+o(1))
$$

when $z$ is near the dominant singularity $\rho$. We provide asymptotic results about the size of the smallest components in random combinatorial structures for the cases $0<\alpha<1$ and any $\beta$, and $\alpha<0$ and $\beta=0$. The particular case $\alpha=0$ and $\beta=1$, the so-called exp-log class, has been treated in previous papers. We also provide similar asymptotic estimates for combinatorial objects with a restricted pattern, that is, when part of its factorization pattern is known. We extend our results to include certain type of integers partitions.

Keywords: decomposable structures; restricted pattern; labeled and unlabeled structures; generating functions; alg-log type

To Philippe for all the lectures and friendship.

\section{Introduction}

A decomposable combinatorial structure consists of simpler objects called components which by themselves can not be further decomposed. For example, permutations decompose into cycles, graphs into connected components, and polynomials over finite fields into irreducible factors.

We assume that the component generating function $C(z)$ is of alg-log type, that is, near the singularity $\rho, C(z)$ behaves like

$$
c+d(1-z / \rho)^{\alpha}\left(\ln \frac{1}{1-z / \rho}\right)^{\beta}(1+o(1)) .
$$

1365-8050 (c) 2010 Discrete Mathematics and Theoretical Computer Science (DMTCS), Nancy, France 
A precise definition is given in the following sections. We call $\alpha$ the algebraic exponent and $\beta$ the logarithmic exponent.

In the particular important case when $\alpha=0$ and $\beta=1$, the components are called of logarithmic type, and the objects are said to be in the exp-log class. Several results have been proven for these objects. Flajolet and Soria [6] introduced the exp-log class of combinatorial structures and studied the behavior of the number of components for structures in this class. Chapter 5 in Gourdon's thesis [8] studies the size of the $r$ th largest component for objects of the exp-log type. The problem of the distribution of the $r$ th smallest component in this class has been studied by Panario and Richmond [12. Arratia, Barbour and Tavaré [1] also studied decomposition of random combinatorial structures in the exp-log class from a probabilistic standpoint. Finally, the study of objects in the exp-log class with a restricted pattern combined with information on the smallest components' sizes was done by Dong et al. [2, 3].

The class of generic alg-log component generating functions (that is, not for the particular exp-log case) was introduced by Flajolet and Soria [7 when studying the number of components in combinatorial structures. Gourdon in Chapter 6 of his thesis [8] (see also [9]) study the size of the $r$ th largest components for objects of the alg-log type.

In this paper we focus on components of the alg-log type and treat the cases $0<\alpha<1$ with any $\beta$, and $\alpha<0$ and $\beta=0$. We carry out the asymptotic analysis using two different approaches: the first case is treated using singularity analysis; the case $\alpha<0, \beta=0$ is done using the saddle point method (Hayman's approach).

We now give the structure and main results of this paper. Generating functions are the main combinatorial technique that we consider. We use exponential generating functions for labeled objects and ordinary generating functions for unlabeled objects composed using the multi-set construction. In Section 2, we review the generating functions of labeled and unlabeled decomposable structures with a restricted pattern, as well as the definition of components of the alg-log type. We also provide in that section most of the notation needed for the rest of the paper. In Section 3, we use similar techniques to Flajolet and Odlyzko [4, and Flajolet and Sedgewick's book [5], to prove our main results about number of objects with restricted pattern and algebraic exponent $0<\alpha<1$ in the labeled and unlabeled case, respectively (Theorem 3.1 and Theorem 3.2). We also provide the respective probabilities (Corollary 3.1 and Corollary 3.3. We give the probability that we have a restricted pattern and the $r$ th smallest component size is bigger than a value $k$ and for both labeled and unlabeled objects (Theorem 3.3 and Theorem 3.4). As important corollaries, we get the particular cases when there are no restricted patterns (Corollary 3.5 and Corollary 3.6. In Section 4, we use the saddle point method to obtain similar studies to the ones in the previous section but for negative algebraic exponent (Theorem 4.1. Theorem 4.2 and Theorem 4.3). In Section 5 , we give examples of our results. Section 6 extends our results of Section 4 to include certain type of restricted integers partitions. Finally, in Section 7 , we state some open problems for further research.

\section{Background}

We recall the following definition of restricted pattern from [2].

Definition 2.1 The restricted pattern of an object of size $n$ is a mapping $S: J \mapsto \mathbb{N}$, where $J$ is a set of components' sizes, $\mathbb{N}$ is the set of nonnegative integers, and $S(j)$ is the number of 
components of size $j$. If we do not have a restricted pattern, $J$ is the empty set. For convenience we also use the notation

$$
S=\left[\prod_{j \in J} j^{S(j)}\right]
$$

We emphasize that we want to count objects such that the components with sizes outside $J$ may appear any number of times but there are exactly $S(j)$ components of size $j$ for each $j \in J$.

Throughout this paper, we use $C(z)$ to denote the generating function for the components. In the labeled case, we have $C(z)=\sum_{k \geq 1} C_{k} \frac{z^{k}}{k !}$. In the unlabeled case we have $C(z)=\sum_{k \geq 1} C_{k} z^{k}$. We also use $C(z ; J)$ to denote the generating function for the components whose sizes are in the given set $J$. So $C(z ; J)=\sum_{j \in J} C_{j} \frac{z^{j}}{j !}$ for the labeled case and $C(z ; J)=\sum_{j \in J} C_{j} z^{j}$ for the unlabeled case.

For convenience we shall use the following notation

$$
\mathscr{S}= \begin{cases}\prod_{j \in J}\left(\frac{C_{j}}{j !}\right)^{S(j)} \frac{1}{S(j) !} & \text { for labeled case } \\
\prod_{j \in J}\left(\begin{array}{c}
C_{j}+S(j)-1 \\
S(j)
\end{array}\right) & \text { for unlabeled case. }\end{cases}
$$

We also let $m^{\prime}=\sum_{j \in J} j S(j)$ denote the size of the restricted pattern, and $m=n-m^{\prime}$ be the total size of the unrestricted components. The following simple lemma can be found in [2].

Lemma 2.1 Let $S: J \mapsto \mathbb{N}$ be a given restricted pattern and $F(z ; S)$ be the generating function of structures with the restricted pattern $S$. For the labeled case, we have

$$
F(z ; S)=\mathscr{S} z^{m^{\prime}} \exp (C(z)-C(z ; J)) .
$$

For the unlabeled case, we have

$$
F(z ; S)=\mathscr{S} z^{m^{\prime}} \exp \left(\sum_{k \geq 1} \frac{C\left(z^{k}\right)-C\left(z^{k} ; J\right)}{k}\right) .
$$

We observe that $F(z, \emptyset)=F(z)$.

In the following we give the definition of component generating function of the alg-log type.

Definition 2.2 Let

$$
\Delta_{\rho}(\nu, \theta)=\{z:|z| \leq \rho(1+\nu), z \neq \rho,|\operatorname{Arg}(z-\rho)| \geq \theta\}
$$

for some constants $\rho>0, \nu>0$, and $0<\theta<\pi / 2$ (see figure). We say that $C(z)$ is of alg-log type at singularity $\rho$ with algebraic exponent $\alpha$ and logarithmic exponent $\beta$, if $C(z)$ is analytic in $\Delta_{\rho}(\nu, \theta)$, and for some constants $c$ and $d$,

$$
C(z)=c+d(1-z / \rho)^{\alpha}\left(\ln \frac{1}{1-z / \rho}\right)^{\beta}(1+o(1))
$$

as $z \rightarrow \rho$ in $\Delta_{\rho}(\nu, \theta)$. 


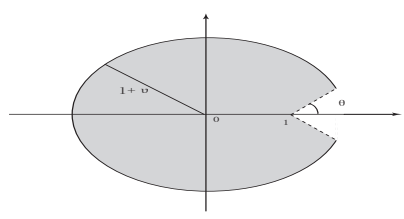

Fig. 1: $\Delta_{1}(\nu, \theta)$

\section{Alg-log components with positive algebraic exponent}

In this section we treat the case $0<\alpha<1$ and any $\beta$. We split the study in restricted patterns in the labeled case, restricted patterns in the unlabeled case and $r$ th smallest component sizes.

\subsection{Labeled case}

Since $0<\alpha<1$, we have from (1) and (3)

$$
F(z, \emptyset)=e^{c}+d e^{c}(1-z / \rho)^{\alpha}\left(\ln \frac{1}{1-z / \rho}\right)^{\beta}(1+o(1))
$$

as $z \rightarrow \rho$ in $\Delta_{\rho}(\nu, \theta)$. It follows from (3) and Flajolet and Odlyzko's transfer theorem [4 that

$$
\frac{C_{n}}{n !} \sim \frac{d}{\Gamma(-\alpha)} \rho^{-n} n^{-\alpha-1}(\ln n)^{\beta},
$$

and

$$
\left[z^{n}\right] F(z ; \emptyset) \sim \frac{d e^{c}}{\Gamma(-\alpha)} \rho^{-n} n^{-\alpha-1}(\ln n)^{\beta} .
$$

Theorem 3.1 Assume $0<\alpha<1, \hat{j}=\max \{j: j \in J\}=O(m / \ln m)$, and

$$
\sum_{j \in J} j^{-\alpha}(\ln j)^{\beta}=o\left(m^{1-\alpha}(\ln m)^{\beta-1}\right) .
$$

Then, as $m \rightarrow \infty$,

$$
\left[z^{n}\right] F(z ; S) \sim d e^{c} \frac{\mathscr{S}}{\Gamma(-\alpha)} \rho^{-m} m^{-\alpha-1}(\ln m)^{\beta} \exp (-C(\rho ; J)),
$$

where the asymptotics is uniform in all $S$.

Proof: 
We shall use the Cauchy integration formula. We choose a contour similar to the one in [4]. Let $\gamma=\gamma_{1} \cup \gamma_{2} \cup \gamma_{3} \cup \gamma_{4}$ be positively oriented, where

$$
\begin{aligned}
& \gamma_{1}=\{z:|z-1|=1 / m, \theta<\operatorname{Arg}(z-1)<2 \pi-\theta\} \\
& \gamma_{2}=\{z: 1 / m \leq|z-1| \leq E \ln m / m, \operatorname{Arg}(z-1)=\theta\} \\
& \gamma_{3}=\{z:|z|=1+3 \ln m / m, \theta<\operatorname{Arg}(z-1)<2 \pi-\theta\} \\
& \gamma_{4}=\{z: 1 / m \leq|z-1| \leq E \ln m / m, \operatorname{Arg}(z-1)=2 \pi-\theta\}
\end{aligned}
$$

where the real number $E$ satisfies

$$
\left|1+E \frac{\ln m}{m} e^{i \theta}\right|=1+3 \ln m / m
$$

Then we have

$$
\left[z^{n}\right] F(z ; S)=\rho^{-n}\left[z^{n}\right] F(\rho z ; S)=\rho^{-m} \mathscr{S} \frac{1}{2 \pi i} \int_{\gamma} f(z) d z
$$

where

$$
f(z)=z^{-(m+1)} \exp (C(\rho z)-C(\rho z ; J)) .
$$

Since $|z| \leq 1+3 \ln m / m$ and $\hat{j}=O(m / \ln m)$, we have

$$
\left|z^{j}\right| \leq\left(1+3 \frac{\ln m}{m}\right)^{j}=1+O(j \ln m / m)=O(1)
$$

We have from (4)

$$
\sum_{j \in J} C_{j} \frac{\rho^{j}}{j !}=O(1)
$$

Hence, when $|z| \leq 1+3 \ln m / m$,

$$
\sum_{j \in J} C_{j} \frac{(\rho z)^{j}}{j !}=O(1) .
$$

Applying (3) and Taylor expansion, on the contour $\gamma$, we obtain

$$
\begin{aligned}
f(z)= & z^{-(m+1)} \exp \left(c+d(1-z)^{\alpha}\left(\ln \frac{1}{1-z}\right)^{\beta}(1+o(1))-\sum_{j \in J} C_{j} \frac{(\rho z)^{j}}{j !}\right) \\
= & \exp \left(c-\sum_{j \in J} C_{j} \frac{\rho^{j}}{j !}\right) z^{-(m+1)} \\
& \times \exp \left(d(1-z)^{\alpha}\left(\ln \frac{1}{1-z}\right)^{\beta}(1+o(1))+\sum_{j \in J} C_{j} \frac{\rho^{j}}{j !}\left(1-z^{j}\right)\right) \\
= & \exp \left(c-\sum_{j \in J} C_{j} \frac{\rho^{j}}{j !}\right) z^{-(m+1)}\left(1+g(z, J)+O\left(g^{2}(z, J)\right)\right)
\end{aligned}
$$


where

$$
g(z, J)=d(1-z)^{\alpha}\left(\ln \frac{1}{1-z}\right)^{\beta}(1+o(1))+\sum_{j \in J} C_{j} \frac{\rho^{j}}{j !}\left(1-z^{j}\right) .
$$

In the last line, we can expand the exponential function because of $(7),(8)$ and $0<\alpha<1$.

Substituting (9) for $f(z)$ in (6), we obtain

$$
\begin{aligned}
{\left[z^{n}\right] F(z ; S) } & =\rho^{-m} \mathscr{S} \exp \left(c-\sum_{j \in J} C_{j} \frac{\rho^{j}}{j !}\right) \frac{1}{2 \pi i} \int_{\gamma} z^{-(m+1)}\left(1+g(z, J)+O\left(g^{2}(z, J)\right)\right) d z \\
= & \rho^{-m} \mathscr{S} \exp \left(c-\sum_{j \in J} C_{j} \frac{\rho^{j}}{j !}\right) \\
& \times \frac{1}{2 \pi i}\left(\int_{\gamma} z^{-(m+1)} d z+\int_{\gamma} z^{-(m+1)} g(z, J) d z+\int_{\gamma} z^{-(m+1)} O\left(g^{2}(z, J)\right) d z\right) \\
= & \rho^{-m} \mathscr{S} \frac{\exp \left(c-\sum_{j \in J} C_{j} \frac{\rho^{j}}{j !}\right)}{2 \pi i}\left(\int_{\gamma} z^{-(m+1)} g(z, J) d z+\int_{\gamma} z^{-(m+1)} O\left(g^{2}(z, J)\right) d z\right),
\end{aligned}
$$

where the term $\int_{\gamma} z^{-(m+1)} d z$ in the second line is 0 .

In the following, we first discuss the integral $\int_{\gamma} z^{-(m+1)} g(z, J) d z$. Then, we show that the second integral $\int_{\gamma} z^{-(m+1)} O\left(g^{2}(z, J)\right) d z$ is negligible compared with $\int_{\gamma} z^{-(m+1)} g(z, J) d z$.

Case I.a: Estimation of $\int_{\gamma} z^{-(m+1)} g(z, J) d z$ on $\gamma_{1} \cup \gamma_{2} \cup \gamma_{4}$ :

Let $z=1+\frac{w}{m}$. From the definition of $\gamma_{1}, \gamma_{2}$, and $\gamma_{4}$, we notice that $w=O(\ln m)$. Since

$$
z^{j}=\left(1+\frac{w}{m}\right)^{j}=1+O(j w / m)
$$

applying 44 and $\sum_{j \in J} \frac{\left(\ln j^{\beta}\right.}{j^{\alpha}}=o\left(m^{1-\alpha}(\ln m)^{\beta-1}\right)$, we get

$$
\begin{aligned}
\sum_{j \in J} C_{j} \frac{\rho^{j}}{j !}\left(1-z^{j}\right) & =\sum_{j \in J} C_{j} \frac{\rho^{j}}{j !} O\left(\frac{j w}{m}\right)=O\left(\frac{w}{m}\right) \sum_{j \in J} j C_{j} \frac{\rho^{j}}{j !} \\
& =O\left(\frac{w}{m} \sum_{j \in J} \frac{(\ln j)^{\beta}}{j^{\alpha}}\right)=o\left(\frac{(\ln m)^{\beta}}{m^{\alpha}}\right) .
\end{aligned}
$$

Hence,

$$
\begin{aligned}
g(z, J) & =d\left(-\frac{w}{m}\right)^{\alpha}\left(\ln \left(-\frac{m}{w}\right)\right)^{\beta}(1+o(1))+\sum_{j \in J} C_{j} \frac{\rho^{j}}{j !}\left(1-z^{j}\right) \\
& =\frac{d(\ln m)^{\beta}}{m^{\alpha}}(-w)^{\alpha}(1+o(1)),
\end{aligned}
$$


where the $o(1)$ is uniform as $m \rightarrow \infty$ for $w$ on the contour $\gamma_{1} \cup \gamma_{2} \cup \gamma_{4}$.

Let $\gamma^{\prime}=\gamma_{1}^{\prime}+\gamma_{2}^{\prime}+\gamma_{4}^{\prime}$ where

$$
\begin{aligned}
\gamma_{1}^{\prime} & =\{w:|w|=1, \theta<\operatorname{Arg}(w)<2 \pi-\theta\} \\
\gamma_{2}^{\prime} & =\{w: 1 \leq|w| \leq E \ln m, \operatorname{Arg}(w)=\theta\} \\
\gamma_{4}^{\prime} & =\{w: 1 \leq|w| \leq E \ln m, \operatorname{Arg}(w)=2 \pi-\theta\} .
\end{aligned}
$$

Then

$$
\begin{aligned}
& \frac{1}{2 \pi i} \int_{\gamma_{1} \cup \gamma_{2} \cup \gamma_{4}} z^{-(m+1)} g(z, J) d z \\
= & \frac{1}{2 \pi i m} \int_{\gamma^{\prime}} \frac{d(\ln m)^{\beta}}{m^{\alpha}}(-w)^{\alpha}(1+o(1))\left(1+\frac{w}{m}\right)^{-(m+1)} d w \\
= & \frac{1}{2 \pi i m} \int_{\gamma^{\prime}} \frac{d(\ln m)^{\beta}}{m^{\alpha}}(-w)^{\alpha} e^{-w}(1+o(1)) d w \\
= & \frac{d(\ln m)^{\beta}}{2 \pi i m^{1+\alpha}} \int_{\gamma^{\prime}}(-w)^{\alpha} e^{-w} d w+o\left(\frac{(\ln m)^{\beta}}{m^{1+\alpha}} \int_{\gamma^{\prime}}(-w)^{\alpha} e^{-w} d w\right) .
\end{aligned}
$$

Applying the Gamma function we obtain

$$
\frac{1}{2 \pi i} \int_{\gamma_{1} \cup \gamma_{2} \cup \gamma_{4}} z^{-(m+1)} g(z, J) d z \sim \frac{(\ln m)^{\beta}}{m^{1+\alpha}} \frac{d}{\Gamma(-\alpha)} .
$$

Case I.b: Estimation of $\int_{\gamma} z^{-(m+1)} g(z, J) d z$ on $\gamma_{3}$ :

Let $z=(1+3 \ln m / m) e^{i t}$ where $\theta<t<2 \pi-\theta$. Using (8) and the definition of alg-log type, we obtain

$$
|g(z, J)|=O(1)
$$

We also have

$$
\left|z^{-(m+1)}\right|=\left|(1+3 \ln m / m)^{-(m+1)}\right|=O\left(1 / m^{3}\right) .
$$

Therefore,

$$
\frac{1}{2 \pi i} \int_{\gamma_{3}} z^{-(m+1)} g(z, J) d z=O\left(m^{-3}\right) .
$$

Since we have $0<\alpha<1$, the integral on $\gamma_{3}$ is negligible compared with the integral on $\gamma_{1} \cup \gamma_{2} \cup \gamma_{4}$. Case II.a: Estimation of $\int_{\gamma} z^{-(m+1)} O\left(g^{2}(z, J)\right) d z$ on $\gamma_{1} \cup \gamma_{2} \cup \gamma_{4}$ :

Let $z=1+\frac{w}{m}$. From 10 ,

$$
g^{2}(z, J)=\frac{d^{2}(\ln m)^{2 \beta}}{m^{2 \alpha}}(-w)^{2 \alpha}(1+o(1))
$$

Using arguments similar to those used to derive (11), we have

$$
\int_{\gamma_{1} \cup \gamma_{2} \cup \gamma_{4}} z^{-(m+1)} g^{2}(z, J) d z=O\left(\frac{(\ln m)^{2 \beta}}{m^{1+2 \alpha}}\right) .
$$


Case II.b: Estimation of $\int_{\gamma} z^{-(m+1)} O\left(g^{2}(z, J)\right) d z$ on $\gamma_{3}$ :

In this case we get

$$
\int_{\gamma_{3}} z^{-(m+1)} g^{2}(z, J) d z=O\left(m^{-3}\right),
$$

which is negligible compared with the integral on $\gamma_{1} \cup \gamma_{2} \cup \gamma_{4}$.

Since $0<\alpha<1$, it is clear that the integral

$$
\rho^{-m} \mathscr{S} \frac{\exp \left(c-\sum_{j \in J} C_{j} \frac{\rho^{j}}{j !}\right)}{2 \pi i} \int_{\gamma} z^{-(m+1)} g(z, J) d z
$$

is the dominant part in $\left[z^{n}\right] F(z ; S)$.

Throughout this paper, a random structure is always chosen uniformly at random from a given family of structures. Hence $P(S, n)=\frac{\left[z^{n}\right] F(z ; S)}{\left[z^{n}\right] F(z ; \emptyset)}$ is the probability that a random decomposable combinatorial structure of size $n$ has a restricted pattern $S$. Using the previous theorem and (5) we immediately obtain the following corollary.

Corollary 3.1 Consider labeled decomposable combinatorial structures whose component generating function is of alg-log type with an algebraic exponent $0<\alpha<1$. Suppose

$$
\hat{j}=\max \{j ; j \in J\}=O(m / \ln m),
$$

and

$$
\sum_{j \in J} \frac{(\ln j)^{\beta}}{j^{\alpha}}=o\left(m^{1-\alpha}(\ln m)^{\beta-1}\right)
$$

We have, as $m \rightarrow \infty$,

$$
P(S, n) \sim\left(\frac{n}{m}\right)^{1+\alpha}\left(\frac{\ln m}{\ln n}\right)^{\beta} \prod_{j \in J} \frac{1}{S(j) !}\left(C_{j} \rho^{j} / j !\right)^{S(j)} e^{-C_{j} \rho^{j} / j !} .
$$

For a restricted pattern of small size, we have the following independence result.

Corollary 3.2 Suppose, in addition to the conditions in Corollary 3.1, the restricted pattern

$$
S=\left[\prod_{j \in J} j^{S(j)}\right]
$$

also satisfies $\sum_{j \in J} j S(j)=o(n)$, that is, $m \sim n$. We have, as $n \rightarrow \infty$,

$$
P(S, n) \sim \prod_{j \in J} P\left(\left[j^{S(j)}\right], n\right) \sim \prod_{j \in J} \frac{1}{S(j) !}\left(C_{j} \rho^{j} / j !\right)^{S(j)} e^{-C_{j} \rho^{j} / j !} .
$$




\subsection{Unlabeled case}

In the unlabeled case, the generating function of decomposable combinatorial structures is

$$
F(z)=F(z, \emptyset)=\exp \left(C(z)+\frac{C\left(z^{2}\right)}{2}+\frac{C\left(z^{3}\right)}{3}+\cdots\right) .
$$

As usual, for the unlabeled case, we assume $0<\rho<1$. Therefore, $\frac{C\left(z^{2}\right)}{2}+\frac{C\left(z^{3}\right)}{3}+\cdots$ has a radius of convergence bigger than $\rho$. As $z \rightarrow \rho$, we have

$$
F(z, \emptyset)=\exp \left(C(z)+r_{0}\right)(1+o(1)) .
$$

where

$$
r_{0}=\sum_{k \geq 2} \frac{C\left(\rho^{k}\right)}{k}
$$

It is easy to see that $F(z, \emptyset)$ is also of alg-log type. Flajolet and Odlyzko's transfer theorem [4] entails

$$
C_{n} \sim \frac{d}{\Gamma(-\alpha)} \rho^{-n} n^{-1-\alpha}(\ln n)^{\beta},
$$

and

$$
\left[z^{n}\right] F(z ; \emptyset) \sim \frac{d e^{c+r_{0}}}{\Gamma(-\alpha)} \rho^{-n} n^{-1-\alpha}(\ln n)^{\beta} .
$$

Theorem 3.2 Suppose $\hat{j}=\max \{j: j \in J\}=O(m / \ln m)$ and

$$
\sum_{j \in J} j^{-\alpha}(\ln j)^{\beta}=o\left(m^{1-\alpha}(\ln m)^{\beta-1}\right) .
$$

Then, as $m \rightarrow \infty$,

$$
\left[z^{n}\right] F(z ; S) \sim d e^{c+r_{0}} \frac{\mathscr{S}}{\Gamma(-\alpha)} m^{-\alpha-1}(\ln m)^{\beta} \rho^{-m} \prod_{j \in J}\left(1-\rho^{j}\right)^{C_{j}} .
$$

Proof: The proof is similar to the labeled case so we only sketch the differences. We use the same contour as in Theorem 3.1 but now

$$
f(z)=\frac{1}{z^{m+1}} \exp \left(\sum_{k \geq 1} \frac{C\left((\rho z)^{k}\right)}{k}-\sum_{k \geq 1} \frac{C\left((\rho z)^{k} ; J\right)}{k}\right) .
$$

Using (14) and (9), for $z \in \gamma$, we get

$$
\begin{aligned}
& \exp \left(C(\rho z)+\frac{C\left((\rho z)^{2}\right)}{2}+\frac{C\left((\rho z)^{3}\right)}{3}+\cdots\right) \\
= & e^{c+r_{0}} \exp (C(\rho z))(1+o(1)) \\
= & e^{c+r_{0}} \exp \left(d(1-z)^{\alpha}\left(\ln \frac{1}{1-z}\right)^{\beta}(1+o(1))\right)(1+o(1)) .
\end{aligned}
$$


The remaining of the proof follows in the same way as in Theorem 3.1 by noting

$$
\exp \left(\sum_{k \geq 1} \rho^{j k} / k\right)=\left(1-\rho^{j}\right)^{-1} \text {. }
$$

Using the previous theorem and (16) we immediately obtain the following corollary.

Corollary 3.3 Consider unlabeled decomposable combinatorial structures whose component generating function is of the alg-log type with an algebraic exponent $0<\alpha<1$. For $\hat{j}=\max \{j ; j \in$ $J\}$, suppose $\hat{j}=O(m / \ln m)$ and

$$
\sum_{j \in J} \frac{(\ln j)^{\beta}}{j^{\alpha}}=o\left(m^{1-\alpha}(\ln m)^{\beta-1}\right) .
$$

We have, as $m \rightarrow \infty$,

$$
P(S, n) \sim\left(\frac{n}{m}\right)^{1+\alpha}\left(\frac{\ln m}{\ln n}\right)^{\beta} \prod_{j \in J}\left(\begin{array}{c}
C_{j}+S(j)-1 \\
S(j)
\end{array}\right) \rho^{j S(j)}\left(1-\rho^{j}\right)^{C_{j}} .
$$

As in the labeled case, we have the following independence result for small patterns.

Corollary 3.4 Suppose, in addition to the conditions in Corollary 3.3, the restricted pattern

$$
S=\left[\prod_{j \in J} j^{S(j)}\right]
$$

also satisfies $\sum_{j \in J} j S(j)=o(n)$, that is, $m \sim n$. We have, as $n \rightarrow \infty$,

$$
P(S, n) \sim \prod_{j \in J} P\left(\left[j^{S(j)}\right], n\right) \sim \prod_{j \in J}\left(\begin{array}{c}
C_{j}+S(j)-1 \\
S(j)
\end{array}\right) \rho^{j S(j)}\left(1-\rho^{j}\right)^{C_{j}} .
$$

\subsection{The rth smallest component size}

Let $X_{n}^{[r]}$ denote the size of the $r$ th smallest component in a random structure of size $n$, and let $X_{n}^{[r]}(S)$ denote the size of the $r$ th smallest component in a random structure of size $n$ having a restricted pattern $S$.

The focus of this section is to derive asymptotic expressions for $P\left(X_{n}^{[r]}(S)>k\right)$ and $P\left(X_{n}^{[r]}>\right.$ $k)$. For convenience, we define the set $N_{k}=\{1,2, \ldots, k\}$ where $k$ is a positive integer and $d(k)=\sum_{j \in J \cap N_{k}} S(j)$. We only need to consider $d(k)<r$ since $P\left(X_{n}^{[r]}(S)>k\right)=0$ when $d(k) \geq r$.

The following simple lemma will be required later.

Lemma 3.1 Let $a$ and $b$ be real constants such that $0<a<1$. Then

$$
\sum_{j=1}^{k} \frac{(\ln j)^{b}}{j^{a}} \sim \frac{1}{1-a} k^{1-a}(\ln k)^{b}, \quad \text { as } \quad k \rightarrow \infty .
$$


Proof: Let $k^{\prime}=\left\lfloor e^{\frac{b}{a}}\right\rfloor+1$. It is easy to check that $\frac{(\ln x)^{b}}{x^{a}}$ is a decreasing function for $x \geq k^{\prime}$. Hence

$$
\int_{k^{\prime}}^{k} \frac{(\ln x)^{b}}{x^{a}} d x+\frac{(\ln k)^{b}}{k^{a}} \leq \sum_{j=k^{\prime}}^{k} \frac{(\ln j)^{b}}{j^{a}} \leq \int_{k^{\prime}}^{k} \frac{(\ln x)^{b}}{x^{a}} d x+\frac{\left(\ln k^{\prime}\right)^{b}}{k^{\prime a}} .
$$

Setting $x=e^{t}$, we have

$$
\begin{aligned}
\int_{k^{\prime}}^{k} \frac{(\ln x)^{b}}{x^{a}} d x & =\int_{\ln k^{\prime}}^{\ln k} t^{b} e^{(1-a) t} d t \\
& =\left.\frac{1}{1-a} e^{(1-a) t} t^{b}\right|_{\ln k^{\prime}} ^{\ln k}-\frac{b}{1-a} \int_{\ln k^{\prime}}^{\ln k} t^{b-1} e^{(1-a) t} d t \\
& =\frac{1}{1-a} k^{1-a}(\ln k)^{b}+O\left(k^{1-a}(\ln k)^{b-1}\right), \quad \text { as } k \rightarrow \infty
\end{aligned}
$$

Now Lemma 3.1 immediately follows from 18 .

\subsubsection{Labeled case}

Theorem 3.3 Let $S: J \mapsto \mathbb{N}$ be a restricted pattern with $\hat{j}=O(m / \ln m)$ and

$$
\sum_{j \in J} j^{-\alpha}(\ln j)^{\beta}=o\left(m^{1-\alpha}(\ln m)^{\beta-1}\right) .
$$

Assume $r-d(k)=O(\ln m)$ and $k=o\left(m(\ln m)^{\frac{1}{\alpha-1}}\right)$. As $m \rightarrow \infty$, we have

$$
P\left(X_{n}^{[r]}(S)>k\right) \sim P(S, n) \exp \left(-C\left(\rho ; N_{k} \backslash J\right)\right) \sum_{j=0}^{r-1-d(k)} \frac{C^{j}\left(\rho ; N_{k} \backslash J\right)}{j !},
$$

where $P(S, n)$ is given in Corollary 3.1 .

Proof: Define the augmented pattern $S^{\star}: J^{\star} \mapsto \mathbb{N}$ where $J^{\star}=J \cup N_{k}$, and $S^{\star}(j)=S(j)$ as $j \in J$, and, for $j \in N_{k} \backslash J, S^{\star}(j)$ is specified below. We note that each structure with restricted pattern $S$ and the $r$ th smallest component with size greater than $k$ corresponds to structures with an augmented pattern $S^{\star}$ such that

$$
\sum_{j \in N_{k} \backslash J} S^{\star}(j) \leq r-1-d(k) .
$$

Hence we have

$$
P\left(X_{n}^{[r]}(S)>k\right)=\sum P\left(S^{\star}, n\right)
$$

where the sum is over all the augmented patterns $S^{\star}$ satisfying 19 .

Let $m^{\star}=n-\sum_{j \in J \cup N_{k}} j S^{\star}(j)$. Since $k=o(m / \ln m)$ and

$$
\sum_{j \in N_{k} \backslash J} S^{\star}(j) \leq r-1-d(k)=O(\ln m),
$$


we have $m^{\star} \sim m$. Hence,

$$
\left(\frac{n}{m^{\star}}\right)^{1+\alpha}\left(\frac{\ln m^{\star}}{\ln n}\right)^{\beta} \sim\left(\frac{n}{m}\right)^{1+\alpha}\left(\frac{\ln m}{\ln n}\right)^{\beta}
$$

Applying $k=o\left(m(\ln m)^{\frac{1}{\alpha-1}}\right)$ and Lemma 3.1 we get

$$
\sum_{j \in N_{k}} \frac{(\ln j)^{\beta}}{j^{\alpha}}=o\left(m^{1-\alpha}(\ln m)^{\beta-1}\right) .
$$

Therefore, each augmented pattern $S^{\star}$ satisfies the requirements of Corollary 3.1. It follows from 20) that

$$
\begin{aligned}
& P\left(X_{n}^{[r]}(S)>k\right) \\
\sim & \sum\left(\frac{n}{m^{\star}}\right)^{1+\alpha}\left(\frac{\ln m^{\star}}{\ln n}\right)^{\beta} \rho^{n-m^{\star}} \exp \left(-C\left(\rho ; J \cup N_{k}\right)\right) \prod_{j \in J \cup N_{k}} \frac{C_{j}^{S^{\star}(j)}}{(j !)^{S^{\star}(j)} S^{\star}(j) !} \\
\sim & \left(\frac{n}{m}\right)^{1+\alpha}\left(\frac{\ln m}{\ln n}\right)^{\beta} \mathscr{S} \rho^{n-m} \exp \left(-C(\rho ; J)-C\left(\rho ; N_{k} \backslash J\right)\right) \sum\left(\prod_{j \in N_{k} \backslash J} \frac{C_{j}^{S^{\star}(j)} \rho^{j S^{\star}(j)}}{(j !)^{S^{\star}(j)} S^{\star}(j) !}\right) \\
\sim & P(S, n) \exp \left(-C\left(\rho ; N_{k} \backslash J\right)\right) \sum\left(\prod_{j \in N_{k} \backslash J} \frac{C_{j}^{S^{\star}(j)} \rho^{j S^{\star}(j)}}{(j !)^{S^{\star}(j)} S^{\star}(j) !}\right),
\end{aligned}
$$

where all the sums are over $S^{\star}$ satisfying 19 . It follows from the multinomial formula

$$
\begin{aligned}
\left(Y_{1}+Y_{2}+\cdots+Y_{k}\right)^{j} & =\sum_{a_{1}+a_{2}+\cdots+a_{k}=j}\left(\begin{array}{c}
j \\
a_{1}, a_{2}, \ldots, a_{k}
\end{array}\right) Y_{1}^{a_{1}} Y_{2}^{a_{2}} \ldots Y_{k}^{a_{k}} \\
& =j ! \sum_{a_{1}+a_{2}+\cdots+a_{k}=j} \prod_{i=1}^{k} \frac{Y_{i}^{a_{i}}}{a_{i} !}
\end{aligned}
$$

that

$$
P\left(X_{n}^{[r]}(S)>k\right) \sim P(S, n) \exp \left(-C\left(\rho ; N_{k} \backslash J\right)\right) \sum_{j=0}^{r-1-d(k)} \frac{C^{j}\left(\rho ; N_{k} \backslash J\right)}{j !}
$$

The following corollary treats the special case when there is no restricted pattern. The corresponding problem for the exp-log class was treated in [12].

Corollary 3.5 Let $r=O(\ln n)$ and $k=o\left(n(\ln n)^{\frac{1}{\alpha-1}}\right)$. As $n \rightarrow \infty$, we have

$$
P\left(X_{n}^{[r]}>k\right) \sim \exp \left(-C\left(\rho ; N_{k}\right)\right) \sum_{j=0}^{r-1} \frac{C^{j}\left(\rho ; N_{k}\right)}{j !}
$$




\subsubsection{Unlabeled case}

In the unlabeled case, we require the singularity point $0<\rho<1$ which is not a required condition in the labeled case.

Theorem 3.4 Let $S: J \mapsto \mathbb{N}$ be a restricted pattern with $\hat{j}=O(m / \ln m)$ and

$$
\sum_{j \in J} \frac{(\ln j)^{\beta}}{j^{\alpha}}=o\left(m^{1-\alpha}(\ln m)^{\beta-1}\right) .
$$

Assume $r-d(k)=O(\ln m)$ and $k=o\left(m(\ln m)^{\frac{1}{\alpha-1}}\right)$. As $m \rightarrow \infty$, we have

$$
\begin{gathered}
P\left(X_{n}^{[r]}(S)>k\right) \sim P(S, n) \exp \left(-\sum_{\ell \geq 1} \frac{C\left(\rho^{\ell} ; N_{k} \backslash J\right)}{\ell}\right) \\
\sum_{j=0}^{r-1-d(k)}\left[z^{j}\right] \exp \left(z C\left(\rho ; N_{k} \backslash J\right)+z^{2} \frac{C\left(\rho^{2} ; N_{k} \backslash J\right)}{2}+\cdots\right),
\end{gathered}
$$

where $P(S, n)$ is given in Corollary 3.3 .

Proof: $\quad$ Let $S^{\star}$ and $m$ be as defined as in the proof of Theorem 3.3 For the same reason as in the labeled case, we have

$$
P\left(X_{n}^{[r]}(S)>k\right)=\sum P\left(S^{\star}, n\right)
$$

where the sum is over all augmented patterns $S^{\star}$ satisfying 19 .

Also we have

$$
\left(\frac{n}{m^{\star}}\right)^{1+\alpha}\left(\frac{\ln m^{\star}}{\ln n}\right)^{\beta} \sim\left(\frac{n}{m}\right)^{1+\alpha}\left(\frac{\ln m}{\ln n}\right)^{\beta},
$$

and each augmented pattern $S^{\star}$ satisfies the requirements of Corollary 3.3 So we can apply Corollary 3.3 to obtain

$$
\begin{aligned}
& P\left(X_{n}^{[r]}(S)>k\right) \\
\sim & \sum\left(\frac{n}{m^{\star}}\right)^{1+\alpha}\left(\frac{\ln m^{\star}}{\ln n}\right)^{\beta} \prod_{j \in J \cup N_{k}}\left(\begin{array}{c}
C_{j}+S^{\star}(j)-1 \\
S^{\star}(j)
\end{array}\right)\left(1-\rho^{j}\right)^{C_{j}} \rho^{n-m^{\star}} \\
\sim & P(S, n) \prod_{j \in N_{k} \backslash J}\left(1-\rho^{j}\right)^{C_{j}} \sum \prod_{j \in N_{k} \backslash J}\left(\begin{array}{c}
C_{j}+S^{\star}(j)-1 \\
S^{\star}(j)
\end{array}\right) \rho^{j S^{\star}(j)},
\end{aligned}
$$

where, as before, the sum is over all $S^{\star}$ satisfying (19).

Using

$$
\left(1-\rho^{i} z\right)^{-C_{i}}=\sum_{\ell \geq 0}\left(\begin{array}{c}
C_{i}+\ell-1 \\
\ell
\end{array}\right) \rho^{i \ell} z^{\ell},
$$


we get

$$
\begin{aligned}
& \sum_{j \in N_{k} \backslash J}\left(\begin{array}{c}
C_{j}+S^{\star}(j)-1 \\
S^{\star}(j)
\end{array}\right) \rho^{j S^{\star}(j)}=\sum_{u=0}^{r-1-d(k)}\left[z^{u}\right] \prod_{j \in N_{k} \backslash J}\left(1-\rho^{j} z\right)^{-C_{j}} \\
= & \sum_{u=0}^{r-1-d(k)}\left[z^{u}\right] \exp \left(-\sum_{j \in N_{k} \backslash J} C_{j} \ln \left(1-\rho^{j} z\right)\right)=\sum_{u=0}^{r-1-d(k)}\left[z^{u}\right] \exp \left(\sum_{i \geq 1} \sum_{j \in N_{k} \backslash J} C_{j} \rho^{i j} z^{i} / i\right) \\
= & \sum_{u=0}^{r-1-d(k)}\left[z^{u}\right] \exp \left(z\left(C\left(\rho ; N_{k}\right)-C\left(\rho ; J \cap N_{k}\right)\right)+z^{2} \frac{C\left(\rho^{2} ; N_{k}\right)-C\left(\rho^{2} ; J \cap N_{k}\right)}{2}+\cdots\right) .
\end{aligned}
$$

To complete the proof we only need to notice that

$$
\prod_{j \in N_{k} \backslash J}\left(1-\rho^{j}\right)^{C_{j}}=\exp \left(-\sum_{\ell \geq 1} \frac{C\left(\rho^{\ell} ; N_{k} \backslash J\right)}{\ell}\right) .
$$

The case when there is no restricted pattern gives the following simple result.

Corollary 3.6 Let $r=O(\ln n)$ and $k=o\left(n(\ln n)^{\frac{1}{\alpha-1}}\right)$. As $n \rightarrow \infty$, we have

$$
P\left(X_{n}^{[r]}>k\right) \sim \exp \left(-\sum_{k \geq 1} \frac{C\left(\rho^{k} ; N_{k}\right)}{k}\right) \sum_{j=0}^{r-1}\left[z^{j}\right] \exp \left(z C\left(\rho ; N_{k}\right)+z^{2} \frac{C\left(\rho^{2} ; N_{k}\right)}{2}+\cdots\right) .
$$

\subsubsection{Expectation of the $r$ th smallest component size}

In this section, we discuss the expectation of the $r$ th smallest component with a given restricted pattern. We use $\hat{d}$ to denote the total number of components in the given restricted pattern, namely $\hat{d}=\sum_{j \in J} S(j)$. If $r \leq \hat{d}$, it is clear that $P\left(X_{n}^{[r]}(S)>k\right)=0$ when $\sum_{j \in J \cap N_{k}} S(j) \geq r$. Hence we have

$$
E\left(X_{n}^{[r]}(S)\right)=\sum_{k=0}^{i^{[r]}-1} P\left(X_{n}^{[r]}(S)>k\right),
$$

where $i^{[r]}$ is the size of the $r$ th component in the pattern $S$.

Theorem 3.5 Let $S: J \mapsto \mathbb{N}$ be a restricted pattern satisfying $\hat{j}=o\left(m(\ln m)^{\frac{1}{\alpha-1}}\right)$ and

$$
\sum_{j \in J} \frac{(\ln j)^{\beta}}{j^{\alpha}}=o\left(m^{1-\alpha}(\ln m)^{\beta-1}\right) .
$$

When $r=\hat{d}+1$, we have in the labeled case

$$
E\left(X_{n}^{[r]}(S)\right) \sim P(S, n) m \exp (-c+C(\rho ; J))
$$


and in the unlabeled case we get, for $r_{0}=\sum_{k \geq 2} C\left(\rho^{k}\right) / k$,

$$
E\left(X_{n}^{[r]}(S)\right) \sim P(S, n) m \exp \left(-c-r_{0}\right) \prod_{j \in J}\left(1-\rho^{j}\right)^{-C_{j}} .
$$

Proof: To estimate the first moment, we use the well-known expression

$$
E\left(X_{n}^{[r]}(S)\right)=\sum_{k \geq 0} P\left(X_{n}^{[r]}(S)>k\right)
$$

We only prove our theorem in the labeled case since the proof is very similar in the unlabeled case.

Let us begin with a special case: there is only one component in addition to the components of the pattern. Namely, we have $\hat{d}+1$ components in total. Thus, the size of the only component not in the pattern is $m$. The probability that an object has exactly $\hat{d}+1$ components is

$$
\begin{aligned}
P\left(X_{n}^{[\hat{d}+1]}(S)=m\right) & =\frac{\mathscr{S} C_{m} / m !}{\left[z^{n}\right] F(z)}=\frac{\mathscr{S} \rho^{-m}(\ln m)^{\beta} m^{-1-\alpha}(d+o(1)) / \Gamma(-\alpha)}{e^{c} \rho^{-n}(\ln n)^{\beta} n^{-1-\alpha}(d+o(1)) / \Gamma(-\alpha)} \\
& =\mathscr{S} \rho^{n-m} e^{-c}\left(\frac{n}{m}\right)^{1+\alpha}\left(\frac{\ln m}{\ln n}\right)^{\beta}(1+o(1)) \\
& \sim P(S, n) e^{-c} \exp (C(\rho ; J)) .
\end{aligned}
$$

Let us now consider small $k$. Setting $t=\max \left(\hat{j}+1,\left\lfloor m(\ln m)^{\frac{2}{\alpha-1}}\right\rfloor\right)$, then $d(t)=\hat{d}, J \subseteq N_{t}$, and $C\left(\rho ; N_{t} \backslash J\right)=C\left(\rho ; N_{t}\right)-C(\rho ; J)$. Applying Theorem 3.3, we obtain

$$
P\left(X_{n}^{[\hat{d}+1]}(S)>t\right) \sim P(S, n) \exp (-c+C(\rho ; J)) .
$$

Clearly, for any $k<m$, we always have

$$
P\left(X_{n}^{[\hat{d}+1]}(S)>k\right) \geq P\left(X_{n}^{[\hat{d}+1]}(S)=m\right)=P(S, n) e^{-c} \exp (C(\rho ; J)) .
$$

Thus, uniformly for $t \leq k \leq m$, we have

$$
P\left(X_{n}^{[\hat{d}+1]}(S)>k\right) \sim P(S, n) e^{-c} \exp (C(\rho ; J)) .
$$

Hence, noting that $t=o\left(m(\ln m)^{\frac{1}{\alpha-1}}\right)$, we get

$$
\begin{aligned}
E\left(X_{n}^{[\hat{d}+1]}(S)\right) & =\sum_{k \geq 0} P\left(X_{n}^{[\hat{d}+1]}(S)>k\right)=\sum_{t \leq k \leq m} P\left(X_{n}^{[\hat{d}+1]}(S)>k\right)+\sum_{0 \leq k<t} P\left(X_{n}^{[\hat{d}+1]}(S)>k\right) \\
& =m P(S, n) \exp (-c+C(\rho ; J))(1+o(1))+O(t P(S, n)) \\
& \sim m P(S, n) \exp (-c+C(\rho ; J)) .
\end{aligned}
$$

In the empty pattern case, let $X_{n}^{[r]}$ be the size of the $r$ th smallest component of a random decomposable combinatorial structure of size $n$. The expectation of $X_{n}^{[1]}$ immediately follows from Theorem 3.5 . 
Corollary 3.7 We have in the labeled case

$$
E\left(X_{n}^{[1]}\right) \sim n e^{-c}
$$

In the unlabeled case, we have

$$
E\left(X_{n}^{[1]}\right) \sim n e^{-c-r_{0}}
$$

\section{Algebraic components with negative algebraic exponent}

In this section, we assume that the component generating function has the following special form

$$
C(z)=d(1-z / \rho)^{-p}+c+o(1) \text { as } z \rightarrow \rho \text { in the open disk }|z|<\rho,
$$

where $c, d$, and $p$ are constants with $p, d>0$, and $C(z)$ is analytic in the open disc $|z|<\rho$. We use $-p, p>0$, instead of $\alpha$ to avoid confusion.

Let $h(z)=d(1-z)^{-p}$. The asymptotic behavior of $\left[z^{n}\right] \exp (h(z))$ has been studied by Wright [16] and Hayman [10]. Hayman showed that $\exp (h(z))$ is admissible in $|z|<1$. Although their results cannot be applied directly to our generating functions involving a restricted pattern, the same saddle point method does apply as well. We note that the contour used here is substantially different from that used in the previous section where the exponent is positive. The contour here is a circle inside the unit disk.

Let $\hat{j}=\max \{j: j \in J\}$. Throughout this section we assume

$$
\hat{j}=o\left(m^{\frac{1}{p+1}}\right), \sum_{j \in J} j^{p-1}=o\left(m^{\frac{p}{6(p+1)}}\right), \text { and } \sum_{j \in J} j^{p}=o\left(m^{\frac{1}{p+1}}\right) .
$$

We note that, when $p \geq 1$, the third condition implies the first condition.

\subsection{Labeled case}

We consider labeled structures in this section. Let $R=R(m)$ be defined by

$$
R h^{\prime}(R)=m, 0<R<1 .
$$

Theorem 4.1 Suppose the pattern $S$ satisfies Condition (22) and let $R$ be defined in (23). Then as $m \rightarrow \infty$,

$$
\left[z^{n}\right] F(z ; S) \sim \frac{1}{\sqrt{2 \pi p(p+1) d}}\left(\prod_{j \in J} \frac{1}{S(j) !}\left(\frac{C_{j}}{j !}\right)^{S(j)} e^{-C_{j} \rho^{j} / j !}\right)(R \rho)^{-m}\left(\frac{m}{p d}\right)^{-\frac{p+2}{2(p+1)}} e^{C(R \rho)} .
$$

Proof: We first note that by (1)

$$
\left[z^{n}\right] F(z ; S)=\prod_{j \in J} \frac{1}{S(j) !}\left(\frac{C_{j}}{j !}\right)^{S(j)} \rho^{-m}\left[z^{m}\right] \exp (h(z)-C(\rho z ; J)+c+o(1)) .
$$


Using the Cauchy integral formula, we obtain

$$
\begin{aligned}
& {\left[z^{m}\right] \exp (h(z)-C(\rho z ; J)+c+o(1)) } \\
= & \frac{1}{2 \pi} R^{-m} \int_{-\pi}^{\pi} \exp \left(h\left(R e^{i \theta}\right)-C\left(R \rho e^{i \theta} ; J\right)-i m \theta+c+o(1)\right) d \theta .
\end{aligned}
$$

It follows from (23) and Lagrange inversion that

$$
1-R=\sum_{k \geq 1} \frac{1}{k}\left(\begin{array}{c}
\frac{1}{p+1} \\
k-1
\end{array}\right)(-1)^{k-1}\left(\frac{p d}{m}\right)^{\frac{k}{p+1}}=\left(\frac{p d}{m}\right)^{\frac{1}{p+1}}+O\left(\left(\frac{1}{m}\right)^{\frac{2}{p+1}}\right) .
$$

As in [10], we define

$$
b(R)=R h^{\prime}(R)+R^{2} h^{\prime \prime}(R) \sim p(p+1) d\left(\frac{m}{d p}\right)^{\frac{p+2}{p+1}} .
$$

Let

$$
\delta=m^{-\frac{1}{p+1}-\frac{5 p}{12(p+1)}}
$$

For $\delta \leq|\theta| \leq \pi$, we have

$$
\left|1-R e^{i \theta}\right|=\left(1+R^{2}-2 R \cos \theta\right)^{1 / 2} \geq\left(1+R^{2}-2 R \cos \delta\right)^{1 / 2}=\left|1-R e^{i \delta}\right| .
$$

Hence, we have

$$
\left|1-R e^{i \theta}\right|^{-p} \leq\left|1-R e^{i \delta}\right|^{-p}=\left|1-2 R \cos \delta+R^{2}\right|^{-p / 2} .
$$

Using the Taylor expansion for $\cos \delta$ we obtain

$$
\left|1-R e^{i \theta}\right|^{-p} \leq(1-R)^{-p}-\frac{p}{2} R(1-R)^{-p-2} \delta^{2}+O\left(\delta^{4}\right) .
$$

We observe that

$$
(1-R)^{-p-2} \delta^{2} \sim\left(\frac{p d}{m}\right)^{-\frac{p+2}{p+1}} m^{-\frac{2}{p+1}-\frac{5 p}{6(p+1)}}=A m^{\frac{p}{6(p+1)}},
$$

for some positive constant $A$. Therefore, we have

$$
\left|\exp \left(h\left(R e^{i \theta}\right)\right)\right| \leq \exp \left(h(R)-A m^{\frac{p}{6(p+1)}}\right),
$$

as $m \rightarrow \infty$, uniformly for $\delta \leq|\theta| \leq \pi$.

For $|\theta| \leq \delta$, we have

$$
\begin{aligned}
h\left(R e^{i \theta}\right) & =d(1-R)^{-p}+i p d R(1-R)^{-p-1} \theta-\frac{b(R)}{2} \theta^{2}+O\left((1-R)^{-p-3} \theta^{3}\right) \\
& =d(1-R)^{-p}+i p d R(1-R)^{-p-1} \theta-\frac{b^{2}(R)}{2} \theta^{2}+o(1) .
\end{aligned}
$$


We now break the integral 25) into the following two parts:

$$
I_{1}=\int_{-\delta}^{\delta} \exp \left(h\left(R e^{i \theta}\right)-C\left(R \rho e^{i \theta} ; J\right)-i m \theta+c+o(1)\right) d \theta,
$$

and

$$
I_{2}=\int_{|\theta| \geq \delta} \exp \left(h\left(R e^{i \theta}\right)-C\left(R \rho e^{i \theta} ; J\right)-i m \theta+c+o(1)\right) d \theta .
$$

Using (4) with $\alpha=-p$, by (22) and (23), and for $|\theta| \leq \delta$, we have

$$
\begin{gathered}
C\left(R \rho e^{i \theta} ; J\right)=\sum_{j \in J} \frac{C_{j}}{j !}(R \rho)^{j} e^{i j \theta}=\sum_{j \in J} \frac{C_{j}}{j !} \rho^{j}\left(1-\left(\frac{p d}{m}\right)^{\frac{1}{p+1}}+O\left(m^{-\frac{2}{p+1}}\right)\right)^{j}(1+O(j \delta)) \\
=C(\rho ; J)+\sum_{j \in J} \frac{j C_{j} \rho^{j}}{j !} O\left(m^{-\frac{1}{p+1}}\right)=C(\rho ; J)+O\left(m^{-\frac{1}{p+1}}\right) \sum_{j \in J} j^{p}=C(\rho ; J)+o(1) .
\end{gathered}
$$

Hence, under Condition 22, we have

$$
\begin{aligned}
I_{1} & =\int_{-\delta}^{\delta} \exp \left(d(1-R)^{-p}-\frac{b(R)}{2} \theta^{2}-C(\rho ; J)+c+o(1)\right) d \theta \\
& \sim \sqrt{\frac{2 \pi}{b(R)}} \exp (C(R \rho)-C(\rho ; J)) .
\end{aligned}
$$

We now show that $I_{2}$ is negligible compared with $I_{1}$. From (27), we have

$$
\left|I_{2}\right|=O\left(\exp \left(C(R \rho)-A m^{\frac{p}{6(p+1)}}+C(\rho ; J)\right)\right)=o\left(\frac{\exp (C(R \rho)-C(\rho ; J))}{\sqrt{b(R)}}\right),
$$

where we used the fact

$$
C(\rho ; J)=O\left(\sum_{j \leq \hat{j}} j^{p-1}\right)=O\left((\hat{j})^{p}\right)=o\left(m^{\frac{p}{\overline{6(p+1)}}}\right) .
$$

Now Theorem 4.1 follows from 24 and 25.

When

$$
n-m=o\left(n^{\frac{1}{p+1}}\right)
$$

it follows from $(26)$ that

$$
(R(m))^{m} \sim(R(n))^{n}
$$

since

$$
\begin{aligned}
(R(m))^{m}= & \exp \left(m\left(-\left(\frac{p d}{m}\right)^{\frac{1}{p+1}}+O\left(m^{-\frac{2}{p+1}}\right)\right)\right) \\
= & \exp \left(\left(n+o\left(n^{\frac{1}{p+1}}\right)\right)-(p d)^{\frac{1}{p+1}}\left(n^{-\frac{1}{p+1}}\left(1+o\left(n^{-\frac{p}{p+1}}\right)\right)^{-\frac{1}{p+1}}\right)\right. \\
& \left.+O\left(\left(n+o\left(n^{\frac{1}{p+1}}\right)^{-\frac{2}{p+1}}\right)\right)\right) \sim(R(n))^{n} .
\end{aligned}
$$


Hence we also have

$$
\exp (C(R(m) \rho)) \sim \exp (C(R(n) \rho))
$$

and the following corollary holds.

Corollary 4.1 Suppose, in addition to the condition (22), the restricted pattern

$$
S=\left[\prod_{j \in J} j^{S(j)}\right]
$$

also satisfies

$$
\sum_{j \in J} j S(j)=o\left(n^{1 /(p+1)}\right)
$$

Then, as $n \rightarrow \infty$,

$$
P(S, n) \sim \prod_{j \in J} P\left(\left[j^{S(j)}\right], n\right) \sim \prod_{j \in J} \frac{1}{S(j) !}\left(C_{j} \rho^{j} / j !\right)^{S(j)} \exp \left(-C_{j} \rho^{j} / j !\right) .
$$

\subsection{Unlabeled case}

As before we assume $\rho<1$ for the unlabeled case. Using essentially the same argument as for the labeled case, we obtain the following theorem and corollary.

Theorem 4.2 Suppose the pattern $S$ satisfies Condition (22) and let $R$ be defined in (23). Then as $m \rightarrow \infty$,

$$
\left[z^{n}\right] F(z ; S) \sim \frac{1}{\sqrt{2 \pi p(p+1) d}}\left(\prod_{j \in J}\left(\begin{array}{c}
C_{j}+S(j)-1 \\
S(j)
\end{array}\right) e^{-C_{j} \rho^{j}}\right)(R \rho)^{-m}\left(\frac{m}{p d}\right)^{-\frac{p+2}{2(p+1)}} e^{C(R \rho)+r_{0}},
$$

where $r_{0}=\sum_{k \geq 2} C\left(\rho^{k}\right) / k$.

Corollary 4.2 Suppose, in addition to Condition (22), the restricted pattern

$$
S=\left[\prod_{j \in J} j^{S(j)}\right]
$$

also satisfies

$$
\sum_{j \in J} j S(j)=o\left(n^{1 /(p+1)}\right)
$$

Then, as $n \rightarrow \infty$,

$$
P(S, n) \sim \prod_{j \in J} P\left(\left[j^{S(j)}\right], n\right) \sim \prod_{j \in J}\left(\begin{array}{c}
C_{j}+S(j)-1 \\
S(j)
\end{array}\right) \rho^{j S(j)}\left(1-\rho^{j}\right)^{C_{j}} .
$$




\subsection{Smallest component size}

Let $X_{n}^{[r]}$ denote the size of the $r$ th smallest component in a random structure of size $n$. The following theorem is analogous to Theorem 3.3 and Theorem 3.4. Its proof is identical to the proofs of Theorem 3.3 and Theorem 3.4 , and is omitted.

Theorem 4.3 Let $r$ be a constant, $k<n^{\epsilon}$ for some small positive constant $\epsilon<1 /(p+1)$ such that $J=N_{k}$ satisfies Condition (22). Let $R=R(n)$ be defined by

$$
p d R(1-R)^{-p-1}=n .
$$

Then, for labeled case

$$
P\left(X_{n}^{[r]}>k\right) \sim \exp \left(-C\left(\rho ; N_{k}\right)\right) \sum_{j=0}^{r-1} C^{j}\left(\rho ; N_{k}\right) / j !,
$$

and for unlabeled case

$$
P\left(X_{n}^{[r]}>k\right) \sim \exp \left(-\sum_{\ell \geq 1} \frac{C\left(\rho^{\ell} ; N_{k}\right)}{\ell}\right) \sum_{j=0}^{r-1}\left[z^{j}\right] \exp \left(\sum_{\ell=1}^{j} \frac{C\left(\rho^{\ell} ; N_{k}\right) z^{\ell}}{\ell}\right),
$$

where the asymptotics is uniform for all $k$ satisfying the given condition, as $n \rightarrow \infty$.

The above expressions for $P\left(X_{n}^{[r]}>k\right)$ are the same as the ones in Corollaries 3.5 and 3.6

We note that

$$
C\left(\rho ; N_{k}\right)=\left[z^{k}\right](1-z)^{-p-1} \sim k^{p} / \Gamma(p+1) \text { as } k \rightarrow \infty .
$$

Thus

$$
P\left(X_{n}^{[r]}>k\right)=O\left(\exp \left(-k^{p / 2}\right)\right) \text { for } k \rightarrow \infty \text { and } k<n^{\epsilon} .
$$

With a similar argument in the unlabeled case, this immediately implies the following.

Corollary 4.3 Let $r$ be any fixed positive integer. For labeled structures we have

$$
E\left(X_{n}^{[r]}\right) \sim \sum_{k \geq 0} \exp \left(-C\left(\rho ; N_{k}\right)\right) \sum_{j=0}^{r-1} C^{j}\left(\rho ; N_{k}\right) / j !
$$

In particular,

$$
E\left(X_{n}^{[1]}\right) \sim \sum_{k \geq 0} \exp \left(-C\left(\rho ; N_{k}\right)\right)
$$

For unlabeled structures we have

$$
E\left(X_{n}^{[r]}\right) \sim \sum_{k \geq 0} \exp \left(-\sum_{\ell \geq 1} \frac{C\left(\rho^{\ell} ; N_{k}\right)}{\ell}\right) \sum_{j=0}^{r-1}\left[z^{j}\right] \exp \left(\sum_{\ell=1}^{j} \frac{C\left(\rho^{\ell} ; N_{k}\right) z^{\ell}}{\ell}\right) .
$$

In particular,

$$
E\left(X_{n}^{[1]}\right) \sim \sum_{k \geq 0} \exp \left(-\sum_{\ell \geq 1} \frac{C\left(\rho^{\ell} ; N_{k}\right)}{\ell}\right)
$$




\section{Examples}

We now give some examples.

Example 5.1 (Cayley Trees) A Cayley tree is a non-planar rooted labeled tree.

Let $C(z)$ be the exponential generating function of Cayley trees. It is well known [5] that

$$
C(z)=z \exp (C(z)), C_{n}=n^{n-1}, C(z)=1-\sqrt{2(1-e z)}+O(1-e z) .
$$

Thus $C(z)$ is of alg-log type with $\rho=1 / e, \alpha=1 / 2, \beta=0, d=-\sqrt{2}$ and $c=1$.

First, let us consider a restricted pattern

$$
S=\left[2^{s} t^{3}\right], \text { where } s=\lfloor\sqrt{n}\rfloor, t=\lfloor\ln n\rfloor .
$$

By Corollary 3.2, we have

$$
P(S, n) \sim \frac{1}{3 !}\left(C_{t} e^{-t} / t !\right)^{3} \exp \left(-C_{t} e^{-t} / t !\right) \frac{1}{s !}\left(C_{2} e^{-2} / 2 !\right)^{s} \exp \left(-C_{2} e^{-2} / 2 !\right) .
$$

Noting that

$$
C_{2} / 2 !=1 \text { and } C_{t} e^{-t} / t ! \sim \frac{1}{\sqrt{2 \pi}} t^{-3 / 2} \text { as } t \rightarrow \infty,
$$

we obtain that the probability of a Cayley forest having $s=\lfloor\sqrt{n}\rfloor$ trees of size 2 and 3 trees of size $t=\lfloor\ln n\rfloor$ is given by

$$
P(S, n) \sim \frac{1}{6}(2 \pi)^{-3 / 2}(\ln n)^{-9 / 2} \frac{1}{s !} e^{-2 s} \exp \left(-e^{-2}\right) .
$$

If in addition to the restricted pattern $S$ we want information related to the 5 th $(r=5)$ smallest component size, we get by Theorems 3.3

$$
P\left(X_{n}^{[5]}(S)>k\right) \sim P(S, n) \exp \left(-C\left(\rho ; N_{k} \backslash J\right)\right) \sum_{j=0}^{4-d(k)} \frac{\left(C\left(\rho ; N_{k} \backslash J\right)\right)^{j}}{j !}, \text { for } k=o\left(n(\ln n)^{-2}\right) .
$$

We note here $d(k)=0$ when $k<2$ and $d(k) \geq s$ when $k \geq 2$. Thus $P\left(X_{n}^{[5]}(S)>k\right)=0$ when $k \geq 2$, and

$$
P\left(X_{n}^{[5]}(S)>1\right) \sim P(S, n) \exp (-1 / e) \sum_{j=0}^{4} \frac{e^{-j}}{j !} .
$$

Finally, by Corollary 3.7, the expected size of the smallest subtree is

$$
E\left(X_{n}^{[1]}\right) \sim \frac{n}{e} .
$$

Example 5.2 (Fragmented permutations) Fragmented permutations are defined in Section II.4.2 of [5] as the combinatorial class $\mathscr{F}=\operatorname{SET}\left(\operatorname{SEQ} Q_{\geq 1}(\mathscr{Z})\right)$. They correspond to unordered collections of permutations. The generating function of fragmented permutations is given by $F(z)=$ 
$\exp \left(\frac{z}{1-z}\right)$. The coefficients in this exponential generating function form the sequence A000262 in Sloane's Encyclopedia of Integer Sequences that counts sets of lists.

The component generating function for fragmented permutations is then

$$
C(z)=\frac{z}{1-z}=\frac{1}{1-z}-1,
$$

and so it is of alg-log type with $\alpha=-1, \rho=1, d=1$ and $c=-1$. It follows from Theorem 4.1] (also see Example VIII.7 of [5]) that

$$
\left[z^{n}\right] F(z)=\frac{F_{n}}{n !} \sim \frac{e^{-1 / 2} e^{2 \sqrt{n}}}{2 \sqrt{\pi} n^{3 / 4}}
$$

and that the saddle point is

$$
R=1-\frac{1}{\sqrt{n}}+\frac{1}{2 n}+O\left(n^{-3 / 2}\right)
$$

Let us consider fragmented permutations with the restricted pattern

$$
S=\left[2^{s} t^{3}\right], \text { where } s=\left\lfloor n^{1 / 4}\right\rfloor, t=\lfloor\ln n\rfloor .
$$

Then Corollary 4.1 gives that the probability a random fragmented permutation has this restricted pattern $S$ is

$$
P(S, n) \sim \frac{1}{3 !}\left(C_{t} / t !\right)^{3} \exp \left(-C_{t} / t !\right) \frac{1}{s !}\left(C_{2} / 2 !\right)^{s} \exp \left(-C_{2} / 2 !\right) .
$$

Noting $C_{j}=j$ !, we obtain

$$
P(S, n) \sim \frac{1}{6} \frac{1}{s !} e^{-2}
$$

We also have, using Corollary 4.3

$$
E\left(X_{n}^{[1]}\right) \sim \sum_{k \geq 0} e^{-k}=\frac{e}{e-1} .
$$

\section{Extensions to integer partitions}

Although our theorems do not cover unlabeled structures with $\rho=1$, similar arguments lead to the same type of results for some unlabeled structures with $\rho=1$. We illustrate this with integer partitions.

Let $A=\left\{a_{1}, a_{2}, \ldots\right\}$ be a set of positive integers. We suppose that if any finite subset of $A$ is deleted then the remaining sequence has gcd equal to one. We let $p_{A}(n)$ be the number of solutions of

$$
n=l_{1} a_{1}+l_{2} a_{2}+\cdots+l_{m} a_{m}, \quad l_{i} \geq 0 .
$$

Call each such representation a partition of $n$. There are $l_{1}+l_{2}+\cdots+l_{m}$ components of this partition.

We require that the following limit exists

$$
\lim _{j \rightarrow \infty} \frac{\ln a_{j}}{\ln j} .
$$


The asymptotic behaviour of $p_{A}(n)$ has been studied by Richmond [13] under the above assumptions. Define $r=r(n)$ to be the unique solution (the saddle point condition) to

$$
n=\sum_{j \geq 1} \frac{a_{j}}{e^{r a_{j}}-1}
$$

Let

$$
A_{2}(n)=A_{2}=\sum_{j \geq 1} \frac{a_{j}^{2} e^{r a_{j}}}{\left(e^{r a_{j}}-1\right)^{2}} .
$$

The following result is contained in Theorem 1.1 of 13

$$
p_{A}(n)=\left(2 \pi A_{2}\right)^{-1 / 2} \exp \left(r n-\sum_{j=1}^{\infty}\left(\ln \left(1-e^{-r a_{j}}\right)\right)\right)(1+O(r)) .
$$

In the following we consider a pattern

$$
S=\left[\prod_{j \in J} j^{S(j)}\right]
$$

such that

$$
\sum_{j \in J} j=o\left(n^{1 / 2}\right), \quad|J|=o\left(n^{1 / 4}\right) \quad \text { and } \quad \sum_{j \in J} j S(j)=o\left(n^{1 / 2}\right),
$$

where $|J|$ denotes the number of elements in the set $J$.

As in previous sections, let $m=n-\sum_{j \in J} j S_{j}$. Let $A=\{1,2, \ldots\} \backslash J$. Then $p_{A}(m)$ is the number of partitions of $n$ with the pattern $S$.

We require now the following formulas about inverse Mellin transform (see, for example, Appendix B of [5]. We also note that the last two formulas can be obtained from the first one by integration and differentiation, respectively, with respect to $r$. )

$$
\begin{aligned}
\sum_{j \geq 1} \frac{j}{e^{r j}-1} & =\frac{1}{2 \pi i} \int_{\sigma-i \infty}^{\sigma+i \infty} r^{-t} \Gamma(t) \zeta(t) \zeta(t-1) d t \\
-\sum_{j \geq 1} \ln \left(1-e^{-r j}\right) & =\frac{1}{2 \pi i} \int_{\sigma-i \infty}^{\sigma+i \infty} r^{-t} \Gamma(t) \zeta(t) \zeta(t+1) d t \\
\sum_{j \geq 1} \frac{j^{2} e^{r j}}{\left(e^{r j}-1\right)^{2}} & =\frac{1}{2 \pi i} \int_{\sigma-i \infty}^{\sigma+i \infty} r^{-t} \Gamma(t) \zeta(t-1) \zeta(t-2) d t
\end{aligned}
$$


It follows from the residue theorem (or use correspondence (47) in Appendix B of [5]) that

$$
\begin{aligned}
\sum_{j \geq 1} \frac{j}{e^{r j}-1} & =r^{-2} \zeta(2)+\zeta(0) r^{-1}+O\left(r^{-\varepsilon}\right), \\
-\sum_{j \geq 1} \ln \left(1-e^{-r j}\right) & =r^{-1} \zeta(2)+\zeta(0) \ln (1 / r)+\zeta^{\prime}(0)+o(1), \\
\sum_{j \geq 1} \frac{j^{2} e^{r j}}{\left(e^{r j}-1\right)^{2}} & =2 \zeta(2) r^{-3}+O\left(r^{-2}\right) .
\end{aligned}
$$

Thus condition (28) becomes

$$
\begin{aligned}
n-\sum_{j \in J} j S(j) & =\sum_{j \in A} \frac{j}{e^{r j}-1}=\sum_{j \geq 1} \frac{j}{e^{r j}-1}-\sum_{j \in J} \frac{j}{e^{r j}-1} \\
& =r^{-2} \zeta(2)+\zeta(0) r^{-1}+O\left(r^{-\varepsilon}\right)-\sum_{j \in J} \frac{j}{e^{r j}-1}
\end{aligned}
$$

Using

$$
\sum_{j \in J} \frac{j}{e^{r j}-1}=\frac{1}{r} \sum_{j \in J}(1+O(r j)),
$$

and (30), we obtain

$$
n=\zeta(2) r^{-2}+r^{-1} o\left(n^{-1 / 4}\right)
$$

Thus

$$
\begin{aligned}
\frac{1}{r} & =\sqrt{\frac{n}{\zeta(2)}}\left(1+o\left(n^{-1 / 2}\right)\right), \\
r & =\sqrt{\frac{\zeta(2)}{n}}\left(1+o\left(n^{-1 / 2}\right)\right) .
\end{aligned}
$$

Finally, substituting the above expressions into Equation 29 and noting $m=n+o\left(n^{1 / 2}\right)$, $\zeta(2)=\pi^{2} / 6, \zeta(0)=-1 / 2$, and $\zeta^{\prime}(0)=-\ln \sqrt{2 \pi}$, we obtain

$$
p_{A}(m) \sim p(n) \prod_{j \in J} \frac{\pi j}{\sqrt{6 n}},
$$

where

$$
p(n) \sim \frac{1}{4 n \sqrt{3}} \exp (\pi \sqrt{2 n / 3})
$$

is the total number of partitions of $n$.

Hence the probability that a random partition of $n$ has the restricted pattern $S$ is

$$
\frac{p_{A}(m)}{p(n)} \sim \prod_{j \in J} \frac{\pi j}{\sqrt{6 n}}
$$


The product on $j$ in the above formula shows the contribution of each part in the restricted pattern is asymptotically independent of each other. This is in agreement with Corollary 4.2 by noting here $\rho=1$ and $C_{j}=1$ for all $j$.

Although we consider here in detail only the case $a_{j}=j$, our results extend, for any positive integer $k$, to $a_{j}=j^{k}$, to $a_{j}=F_{j}^{k}$ where $F_{j}$ is the $j$ th Fibonacci number (where one needs an alternate condition stated in [13]), and to $a_{j}=p_{j}^{k}$, where $p_{j}$ denotes the $j$ th prime number (these assumptions are satisfied since $p_{j} \sim j \ln j$ by the prime number theorem). Of course, conditions stated in 30 need to be adjusted accordingly.

\section{Conclusions}

In this paper, we focus on the multi-set construction of decomposable structures with a given restricted pattern where the component generating function are of special alg-log type. We estimate $P\left(X_{n}^{[r]}>k\right)$ for labeled and unlabeled structures. We remark that for all ranges of $\alpha$ and $\beta$ consider in this paper, $P\left(X_{n}^{[r]}>k\right)$ has the same expression among labeled structures; the same result is true among unlabeled structures.

Let $Y_{n}$ be the number of components in a random structure of size $n$. When the component generating function $C(z)$ is of logarithmic type, it is well known [6, 7] that $Y_{n}$ is asymptotically normal with mean and variance proportional to $\ln n$. Also $E\left(X_{n}^{[r]}\right)$ is proportional to $(\ln n)^{r}[12]$.

We note that, for the labeled case,

$$
P\left(Y_{n}=k\right)=\frac{\left[z^{n}\right] C^{k}(z) / k !}{\left[z^{n}\right] F(z, \emptyset)} .
$$

When $C(z)$ is of alg-log type with algebraic exponent $0<\alpha<1$, we have, from (3) and FlajoletOdlyzko's transfer theorem, that

$$
\left[z^{n}\right] C^{k}(z) \sim \frac{k c^{k-1} d}{\Gamma(-\alpha)} \rho^{-n} n^{-\alpha-1}(\ln n)^{\beta} .
$$

Hence

$$
P\left(Y_{n}=k\right) \sim c^{k-1} e^{-c} /(k-1) !
$$

which is a shifted Poisson distribution with constant mean and variance. Similar results hold for unlabeled structures. We have also shown in this case $E\left(X_{n}^{[1]}\right)$ is proportional to $n$.

When $C(z)$ is of alg-log type with algebraic exponent $\alpha=-p<0$, it follows from [16, 17. that $E\left(Y_{n}\right)$ is proportional to $n^{p /(p+1)}$. We have also shown in this paper that for this algebraic exponent $E\left(X_{n}^{[r]}\right)$ is a constant. For integer partitions, it is also known that the expected number of parts in a random partition of $n$ is proportional to $n^{1 / 2}$ [14. Although integer partitions do not fit into our general framework for unlabeled structures because the radius of convergence is not less than 1, we have shown, using the result of [13], that similar results also hold for integer partitions with a restricted pattern.

Further work. So far we have covered the cases $0<\alpha<1$ and any $\beta$; $\alpha=0$ and $\beta=1$; and $\alpha<0$ and $\beta=0$. It seems possible to extend these results to other ranges of $\alpha$ and $\beta$. In addition, it may be possible to relax the conditions on the restricted pattern $S$. 


\section{References}

[1] Arratia,R., Barbour, A.D., And TAvaré,S., Random combinatorial structures and prime factorizations. Notices of American Mathematical Society, 44 (1997), no 8, 903-910.

[2] Dong,L., Gao,Z. and Panario,D. Enumeration of combinatorial decomposable structures with restricted patterns. Annals of Combinatorics, 12 (2008), 353-368.

[3] Dong,L., Gao,Z. and Panario,D. The size of the rth smallest component in decomposable structures with a restricted pattern. Discrete Mathematics and Theoretical Computer Science (2007), 365-384.

[4] Flajolet,P. and Odlyzko,A. Singularity analysis of generating functions. SIAM J. Discrete Math. 3 (2) (1990), 216-240.

[5] Flajolet,P. and Sedgewick,R. Analytic combinatorics. Cambridge University Press, 2009.

[6] Flajolet,P. And Soria,M. Gaussian limiting distribution for the number of components in combinatorial structures. J. Combin. Theory Ser. A 53 (1990), 165-182.

[7] Flajolet,P. And Soria,M. General combinatorial schemas: Gaussian limiting distributions and exponential tails. Discrete Mathematics 114 (1993), 159-180.

[8] Gourdon,X. Combinatoire, algorithmique et géométrie des polynômes. PhD thesis, École Polytechnique, 1996.

[9] Gourdon,X. Largest component in random combinatorial structures. Discrete Mathematics 180 (1998), 185-209.

[10] Hayman,W. A generalization of Stirling's formula. J. Reine Angew. Math. 196 (1956), $67-95$.

[11] Henrici,P. Applied and computational complex analysis. Vol.2, John Wiley, New York (1974).

[12] Panario,D. and Richmond,B. Smallest components in decomposable structures: exp-log class. Algorithmica 29 (2001), 205-226.

[13] Richmond,B. Asymptotic relations for partitions. Journal of Number Theory 7 (1975), 389-405.

[14] Richmond,B. The moments of partitions, II, Acta Arithmetica XXVIII (1975), 229 - 243.

[15] Whittaker,E.T. and Watson,G.N. A course of modern analysis. fourth ed., Cambridge University Press (1927), Reprinted 1973.

[16] Wright,E.M. The coefficients of a certain power series. J. London Math Society 7 (1932), $256-262$.

[17] Wright,E.M. On the coefficients of power series having exponential singularities. II. J. London Math Society 24 (1949), 304-309. 